\title{
FOREWORD
}

\section{Science Based Technologies for Sustainable and Adequate Energy for India}

Energy is one of the key resources for sustaining quality life on the earth planet. Energy, security, affordability, equity and ethics are intertwined aspects which define policies and the choice basket of technologies for a given country. Furthermore, energy, water, food and healthcare need to be considered in a balanced manner to answer the demands of the country.

India, the second most populous country in the world with approx $270 \mathrm{GW}(\mathrm{e})$ installed capacity of electrical power generation, is also one of the fastest growing economies. Hence, the importance of defining energy policies and technologies, with short, medium and long horizon perspectives. It is true that one-fourth of India's present population ( 300 million) has no access to electricity. About two-third of India's installed capacity of electrical power comes from fossil fuel based thermal power plants of various generations and efficiency spectra, with less than one-fifth from hydro-electricity and all other renewable and clean sources (wind, biomass, solar and nuclear). This pattern in the power generation has not seen much change for Indian basket in last three decades. We have large variations in consumption; near zero to approx. $10000 \mathrm{kwh}$ per capita per annum. It is considered prudent that India should aim at an average 2500-3000 kwh per capita per annum to be a developed country with 8-10\% GOP sustained growth in next two decades to alleviate poverty, ensure security of the nation and provide deserved better quality of life to our citizens, with emphasis on sustainability. This aspiration requires the net installed capacity to be approx $800-900 \mathrm{GW}$ (e) by 2035 so as to satisfy quality of life expectations and economic emergence. We need to accord high importance to energy efficient technologies and low $\mathrm{CO}_{2}$ foot print energy generation, transmission and distribution, energy utilisation by industry, transport, housing and indeed all walks of life.
It can be argued that our continued emphasis on fossil fuels is not sustainable with reference to availability of resources and constraints imposed by deteriorating environment quality and climate change.

Various scenarios on energy have been debated though with divergent opinions. It is important that the science and engineering community in the country undertakes a thorough and comprehensive study to comprehend the status and opportunities related to science, technology innovations, education, human resources and management. It is true that despite our plans and predictions of energy targets and roadmaps, energy aspirations have largely remained unrealized. The Science and technology community, with interdisciplinary and cohesive approach must work with industries, bureaucrats and policy makers to realise adequate energy availability, enhancing, security of resources and sustainability. This needs to be done in an expeditious manner.

I congratulate Dr. Baldev Raj, Prof. Indranil Manna and Dr. Kamachi Mudali, with patronage and support of the Indian National Science Academy, for compiling and editing an excellent compendium of articles covering science, technology, materials, innovation and systems integration related to both renewable and non-renewable energy sources with special emphasis on requirements of India. It is heartening that scholars and specialists from academia, industry and R\&D units have contributed with concise and clear perceptions on status of science, technology gaps and possible pathways of pursuits. It is to be appreciated that in the energy domain, from concept to commercialisation, we need a lifecycle of approximately 20-30 years to effect the changes in the energy basket. The experiences and inevitability of long lifecycle demand, posing the challenges, prioritising domains and pathways supported with appropriate human resources and funding, establishing 
synergy and review mechanisms to achieve success with mission mode mindset and the directions of meeting energy aspirations of India.

I trust that this thematic issue of the Proceedings of the Indian National Science Academy will attract interest of students, teachers, researchers, industry and policy makers. The purpose shall be served better when the knowledge in this publication acts as a catalyst for realizing paradigm changes through good collaborations and sustainable and affordable technologies.
I wish the readers a rewarding experience.

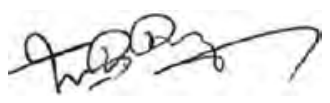

C N R Rao

National Research Professor and Linus Pauling Research Professor

Jawaharlal Nehru Centre for Advanced

Scientific Research 